\title{
Remodelling of the angular collagen fiber distribution in cardiovascular tissues
}

\author{
Niels J. B. Driessen • Martijn A. J. Cox • \\ Carlijn V. C. Bouten · Frank P. T. Baaijens
}

Received: 18 October 2006 / Accepted: 14 January 2007 / Published online: 13 March 2007

(C) Springer-Verlag 2007

\begin{abstract}
Understanding collagen fiber remodelling is desired to optimize the mechanical conditioning protocols in tissue-engineering of load-bearing cardiovascular structures. Mathematical models offer strong possibilities to gain insight into the mechanisms and mechanical stimuli involved in these remodelling processes. In this study, a framework is proposed to investigate remodelling of angular collagen fiber distribution in cardiovascular tissues. A structurally based model for collagenous cardiovascular tissues is extended with remodelling laws for the collagen architecture, and the model is subsequently applied to the arterial wall and aortic valve. For the arterial wall, the model predicts the presence of two helically arranged families of collagen fibers. A branching, diverging hammock-type fiber architecture is predicted for the aortic valve. It is expected that the proposed model may be of great potential for the design of improved tissue engineering protocols and may give further insight into the pathophysiology of cardiovascular diseases.
\end{abstract}

\section{Introduction}

Living tissues show an adaptive response to mechanical load by remodelling their internal structure and morphology. Understanding this response is desired to further optimize the mechanical conditioning protocols for functional tissue engineering of load-bearing cardiovas-

\footnotetext{
N. J. B. Driessen (凶) · M. A. J. Cox · C. V. C. Bouten .

F. P. T. Baaijens

Department of Biomedical Engineering,

Eindhoven University of Technology,

Eindhoven, The Netherlands

e-mail: n.j.b.driessen@tue.nl
}

cular tissues, such as arteries (Niklason et al. 1999) and aortic heart valves (Hoerstrup et al. 2000). In addition, it may give further insight into the effects of mechanical factors on the pathophysiology of cardiovascular diseases, such as atherosclerosis and the formation of aneurysms. Focus in literature has been primarily on the remodelling of the collagen architecture as this is considered to be the main load-bearing component in cardiovascular tissues. Mathematical models can be of great value to gain insight into these remodelling processes and have the capability to infer possible mechanisms involved in these complex processes. Moreover, it is expected that these models can be employed to obtain a more complete understanding of the structurefunction properties of cardiovascular tissues and create the opportunity to incorporate the inhomogeneous collagen architecture in tissues with complex geometries or loading conditions.

Humphrey (1999) studied collagen remodelling in soft connective tissues by considering the deposition and degradation of collagen fibers. Boerboom et al. (2003) and Driessen et al. (2003a,b) modeled mechanically induced collagen fiber remodelling in the aortic valve by assuming that collagen fibers aligned with the strain field and that the collagen content increased with the fiber stretch. With these models, promising results were obtained and the predicted circumferential alignment of the main fiber direction agreed qualitatively with the measured fiber architecture in native aortic valves (Sacks et al. 1997). However, these models also predicted the presence of radially oriented secondary fiber populations whereas these are scarcely present in native valves. In addition, with these models, the typical helical fiber architecture in the arterial wall could not be explained. Therefore, the model was modified to study 
collagen remodelling in the arterial wall and it was hypothesized that the collagen fibers aligned with preferred directions which were situated in between the principal loading directions (Driessen et al. 2004). The predicted fiber directions in the arterial wall represented symmetrically arranged helices and the results agreed qualitatively with data from literature (Rhodin 1980; Finlay et al. 1995; Holzapfel et al. 2002). Subsequently, this framework was applied to study collagen remodelling in the aortic valve (Driessen et al. 2005a) yielding a fiber architecture that represented a branching hammocktype structure which agreed with observations from literature (Sauren 1981). Wilson et al. (2006) employed the same framework to predict the collagen architecture in articular cartilage. However, in these studies only a limited number of fiber directions was employed, whereas measurements demonstrate that multiple fiber directions are present in cardiovascular tissues (Sacks et al. 1998; Holzapfel et al. 2002). In addition, Billiar and Sacks $(2000 \mathrm{a}, \mathrm{b})$ demonstrated that incorporating the angular distribution of collagen fibers is required to accurately describe the complex biaxial mechanical behavior of the aortic valve. Therefore, Driessen et al. (2005b) employed a structurally based constitutive model that contains parameters to incorporate the angular distribution of collagen fibers in cardiovascular tissues and applied it to describe the mechanics of human tissue-engineered heart valve leaflets (Driessen et al. 2007).

The objective of the present study is to model mechanically induced changes in the angular collagen fiber distribution in cardiovascular tissues. In order to accomplish this, the structurally based constitutive model and the hypothesis for collagen remodelling are integrated. We focus on remodelling of the angular fiber distribution only and assume other properties of the collagen architecture (e.g., collagen content, collagen type and collagen cross-links) to be unaffected by the remodelling process. Based on observations of the collagen fiber architecture in uniaxially (e.g., tendons, ligaments) and biaxially loaded tissues (e.g., arterial walls, heart valves), we postulate the following hypotheses. For uniaxial loading conditions, the fibers align with the major loading direction and the dispersity of the fiber distribution decreases (i.e., the fibers become more aligned resulting in a uniaxial fiber distribution). For biaxial loading conditions, on the other hand, the collagen fibers align with preferred directions situated in between the principal loading directions and the dispersity of the distribution increases (i.e., mechanical anisotropy decreases). Finally, for equibiaxial loading conditions, the angular fiber distribution becomes isotropic or uniform. To demonstrate its capabilities, the model is applied to study remodelling of the fiber architecture in the arterial wall and aortic valve. Although we focus primarily on mechanical deformation (i.e., strain) as a stimulus for collagen remodelling, stress-driven remodelling laws are addressed as well, inspired by studies of Olsen et al. (1999), Baek et al. (2006) and Hariton et al. (2006) who consider stress-induced alignment of collagen fibers in wound healing and arterial walls.

\section{Materials and methods}

\subsection{Constitutive law}

The arterial wall and aortic valve are modeled as incompressible composite materials, consisting of an isotropic matrix contribution and an anisotropic fiber contribution (Driessen et al. 2005b). The total Cauchy stress $(\sigma)$ is split into a hydrostatic pressure $(p)$ and an extra stress $(\tau)$ :

$\boldsymbol{\sigma}=-p \boldsymbol{I}+\boldsymbol{\tau}$

The extra stress is written as

$\boldsymbol{\tau}=\hat{\boldsymbol{\tau}}+\sum_{i=1}^{N} \phi_{f}^{i}\left(\psi_{f}^{i}-\vec{e}_{f}^{i} \cdot \hat{\boldsymbol{\tau}} \cdot \vec{e}_{f}^{i}\right) \vec{e}_{f}^{i} \vec{e}_{f}^{i}$

with $\hat{\boldsymbol{\tau}}$ the isotropic matrix stress, $\phi_{f}$ the fiber volume fraction, $\psi_{f}$ the fiber stress, $\vec{e}_{f}$ the current fiber direction and $N$ the number of fiber directions. The matrix material is modeled as a Neo-Hookean material with shear modulus $G$ :

$\hat{\boldsymbol{\tau}}=G(\boldsymbol{B}-\boldsymbol{I})$

The left Cauchy-Green deformation tensor $(\boldsymbol{B})$ is calculated from $\boldsymbol{B}=\boldsymbol{F} \cdot \boldsymbol{F}^{T}$, with $\boldsymbol{F}$ the deformation gradient tensor. The current fiber direction can be calculated from the fiber direction in the undeformed configuration $\left(\vec{e}_{f_{0}}\right)$ :

$\lambda_{f} \vec{e}_{f}=\boldsymbol{F} \cdot \vec{e}_{f_{0}}$

with $\lambda_{f}$ the fiber stretch:

$\lambda_{f}=\sqrt{\vec{e}_{f_{0}} \cdot \boldsymbol{C} \cdot \vec{e}_{f_{0}}}$

and $\boldsymbol{C}=\boldsymbol{F}^{T} \cdot \boldsymbol{F}$ the right Cauchy-Green deformation tensor. The angular fiber distribution can be incorporated into the constitutive model by specifying an appropriate set of fiber contents $\left(\phi_{f}\right)$ and fiber directions $\left(\vec{e}_{f_{0}}\right)$.

In order to incorporate the angular fiber distribution, the fiber directions are defined in a coordinate system spanned by the vectors $\vec{v}_{1}$ and $\vec{v}_{2}$ :

$\vec{e}_{f_{0}}^{i}\left(\gamma_{i}\right)=\cos \left(\gamma_{i}\right) \vec{v}_{1}+\sin \left(\gamma_{i}\right) \vec{v}_{2}$ 
Fig. 1 a Definition of the fiber direction $\vec{e}_{f_{0}}$ and the angle $\gamma$ in a coordinate system spanned by the vectors $\vec{v}_{1}$ and $\vec{v}_{2}$. b Schematic representation of the angular fiber distribution function, indicating the definitions of the main fiber angle $\alpha$, the dispersity $\beta$, and the local directions $\vec{v}_{1}$ and $\vec{v}_{2}$

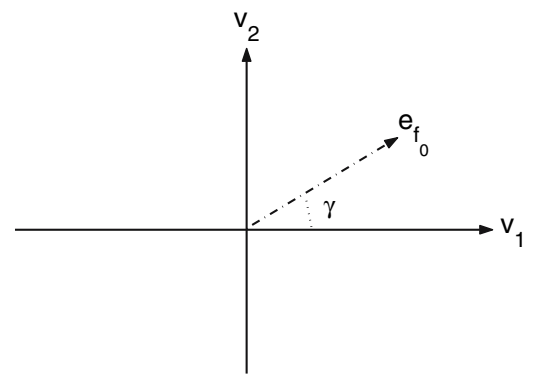

(a)

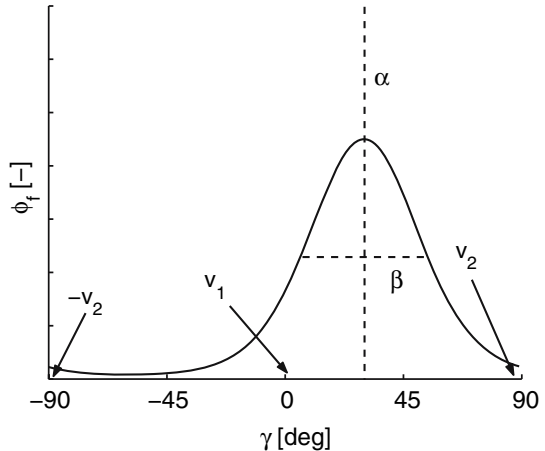

(b) where $\gamma_{i}$ is defined with respect to $\vec{v}_{1}$ in the plane spanned by $\vec{v}_{1}$ and $\vec{v}_{2}$ (Fig. 1a). For the fiber volume fractions a periodic version of the normal probability distribution function is used (Gasser et al. 2006):

$\phi_{f}^{i}\left(\gamma_{i}\right)=A \exp \left[\frac{\cos \left[2\left(\gamma_{i}-\alpha\right)\right]+1}{\beta}\right]$

with $\alpha$ the main fiber angle and $\beta$ the dispersity of the fiber distribution function (Fig. 1b). The scaling factor $A$ is defined such that the total fiber content equals $\phi_{\text {tot }}$. Note that in previous studies (Billiar and Sacks 2000b; Driessen et al. 2005b, 2007) a normal probability or Gaussian function was used, in contrast to Eq. (7). In addition, in previous studies, the fiber distribution parameters were kept constant whereas these are subjected to remodelling in the current study.

\subsection{Collagen fiber remodelling}

It is assumed that the preferred main fiber directions $\left(\alpha_{p}\right)$ are situated in between the local directions $\vec{v}_{1}$ and $\vec{v}_{2}$. In order to specify the value of $\alpha_{p}$, we introduce stimulus functions in the direction of $\vec{v}_{1}$ and $\vec{v}_{2}\left(g_{1}\right.$ and $g_{2}$, respectively). The preferred main fiber direction is then defined as:

$\alpha_{p}=\arctan \left(g_{2} / g_{1}\right)$

The functions $g_{1}$ and $g_{2}$ (Fig. 2a) are still to be specified and indicate the degree of alignment of the preferred main fiber direction with the local direction $\vec{v}_{i}(i=1,2)$. That is, a high value of $g_{i}$ indicates that the preferred main direction is close to $\vec{v}_{i}$. When $g_{1}$ is much larger than $g_{2}$ (i.e, $g_{1}>>g_{2}$ ), then $\alpha_{p} \rightarrow 0^{\circ}$. On the other hand, when $g_{2}$ is much larger than $g_{1}$ (i.e., $g_{1}<<g_{2}$ ), then $\alpha_{p} \rightarrow 90^{\circ}$. Finally, when $g_{1}$ and $g_{2}$ are nearly equal (i.e., $g_{1} \simeq g_{2}$ ), then $\alpha_{p} \rightarrow 45^{\circ}$. To preserve material symmetry, two (preferred) main fiber directions are used in this study which are situated at $\gamma= \pm \alpha$. Note that the assumption for the preferred main fiber direction in Eq. (8) agrees with the previous hypothesis for collagen fiber remodelling (Driessen et al. 2004, 2005a; Wilson et al. 2006) when the directions $\vec{v}_{1}$ and $\vec{v}_{2}$ coincide with the principal loading directions.

For the preferred value of the dispersity $\left(\beta_{p}\right)$, the following expression is used:

$\beta_{p}= \begin{cases}\frac{k}{\left(g_{1} / g_{2}\right)-1} & \text { for } g_{1} \geq g_{2} \\ \frac{k}{\left(g_{2} / g_{1}\right)-1} & \text { for } g_{1}<g_{2}\end{cases}$

with $k$ a scaling factor. Equation (9) implies that when the value of the stimulus function in one direction is much larger than the value in the other direction (i.e., $g_{1}>g_{2}$ or $g_{1}<<g_{2}$ ), then a uniaxial fiber distribution (i.e., $\beta_{p} \rightarrow 0$ ) is obtained. On the other hand, when the values of the stimulus functions in both directions are nearly equal $\left(g_{1} \simeq g_{2}\right)$, then a uniform or isotropic fiber distribution $\left(\beta_{p} \rightarrow \infty\right)$ is obtained (Fig. 2b). The (preferred) dispersities of the two fiber distribution functions are assumed to be equal.

The evolution of the main fiber direction and the dispersity of the fiber distribution function are subsequently modeled by first order rate equations:

$$
\begin{aligned}
\frac{\mathrm{d} \alpha}{\mathrm{d} t} & =\frac{1}{\tau_{a}}\left(\alpha_{p}-\alpha\right) \\
\frac{\mathrm{d} \beta}{\mathrm{d} t} & =\frac{1}{\tau_{b}}\left(\beta_{p}-\beta\right)
\end{aligned}
$$

with $\tau_{a}$ and $\tau_{b}$ the time constants of the remodelling process.

\subsection{Balance equations}

The finite element (FE) method (Bathe 1996) is employed to solve the equations for conservation of momentum:

$\vec{\nabla} \cdot \sigma=\overrightarrow{0}$

and the continuity equation for an incompressible solid:

$J-1=0$ 
Fig. 2 a Definition of the preferred main fiber angle $\alpha_{p}$ in a coordinate system spanned by the vectors $\vec{v}_{1}$ and $\vec{v}_{2}$. The stimulus functions $g_{i}$ in the direction of $\vec{v}_{i}$ are also indicated. b Representation of the preferred dispersity $\beta_{p}$ as a function of the ratio of the stimulus functions $g_{1}$ and $g_{2}$. Note that $\beta_{p} \rightarrow \infty$ for $g_{1} / g_{2}=1$, and $\beta_{p} \rightarrow 0$ for $g_{1} / g_{2} \rightarrow 0$ or $g_{1} / g_{2} \rightarrow \infty$

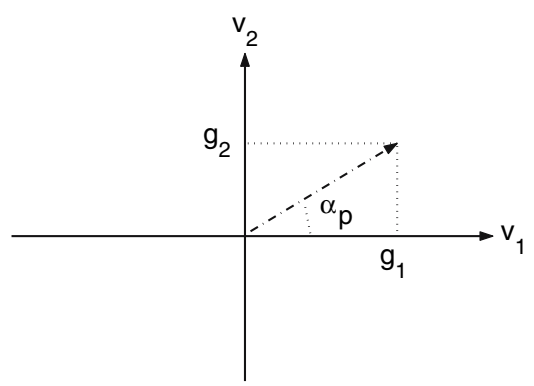

(a)

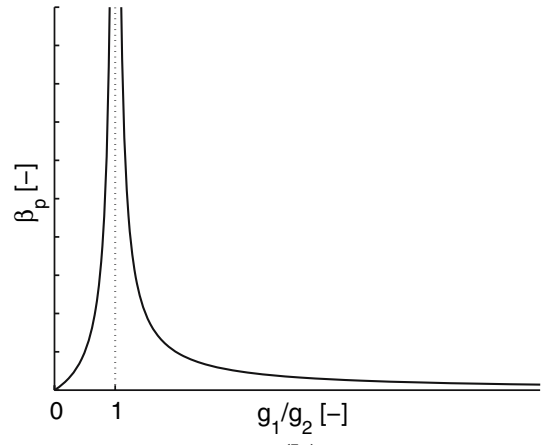

(b) where $J=\operatorname{det}(\boldsymbol{F})$ represents the volume change between the initial and the current configuration. The computational framework is implemented in the software package SEPRAN (Segal 1984) and for further details the reader is referred to Driessen et al. (2005a,b).

\subsection{Geometry and boundary conditions}

\subsubsection{Arterial wall}

The arterial wall is modeled as a single-layered thickwalled cylinder and the FE mesh is shown in Fig. 3a. Geometrical parameters are taken from Holzapfel et al. (2000) and the inner radius and thickness of the cylinder are set to 0.71 and $0.39 \mathrm{~mm}$, respectively. Because of symmetry, only $1 / 4$ of the cylinder is used in the FE simulations. At the symmetry planes, displacements in normal directions are suppressed. At the horizontal surfaces, displacements in the axial direction are prescribed to apply an axial pre-stretch $\left(\lambda_{z}\right)$ of 1.2 and at the inner side of the arterial wall a pressure $\left(p_{i}\right)$ of $13 \mathrm{kPa}$ is applied.

\subsubsection{Aortic valve}

A stented valve geometry is used (Fig. 3b) and because of symmetry only $1 / 6$ of the valve is used in the FE simulations. Geometrical parameters are obtained from Driessen et al. (2005b) and the radius and thickness of the leaflets are set to 12 and $0.46 \mathrm{~mm}$, respectively. At the symmetry surface, displacements in normal directions are suppressed and nodal displacements at the top curve of the fixed edge are set to zero. For simplicity, coaptation of adjacent leaflets is left out of consideration and to model the diastolic transvalvular pressure a uniform pressure of $12 \mathrm{kPa}$ is applied to the leaflets.

\subsection{Parameters}

\subsubsection{Arterial wall}

For the fibers in the arterial wall, the following constitutive equation is used (Holzapfel et al. 2000):

$\psi_{f}= \begin{cases}2 k_{1} \lambda_{f}^{2}\left(\lambda_{f}^{2}-1\right) e^{k_{2}\left(\lambda_{f}^{2}-1\right)^{2}} & \text { for } \lambda_{f} \geq 0 \\ 0 & \text { for } \lambda_{f}<0\end{cases}$

In accordance with previous parameter identification to comply with experimental observations, the fiber material parameters $k_{1}$ and $k_{2}$ are set to $9.47 \mathrm{kPa}$ and 0.81 based on the values for the media layer of a rabbit carotid artery (Holzapfel et al. 2000; Driessen et al. 2005b). The value for the shear modulus of the matrix material $G$ and the total fiber volume fraction $\phi_{\text {tot }}$ are set to $3.0 \mathrm{kPa}$ and 0.5, respectively (Holzapfel et al. 2000; Driessen et al. 2005b). The directions $\vec{v}_{1}$ and $\vec{v}_{2}$ are assumed to coincide with the circumferential and axial direction of the arterial wall, respectively.

\subsubsection{Aortic valve}

For the fibers in the aortic valve, the following stressstretch relationship is assumed:

$\psi_{f}= \begin{cases}k_{1} \lambda_{f}^{2}\left[\mathrm{e}^{k_{2}\left(\lambda_{f}^{2}-1\right)}-1\right] & \text { for } \lambda_{f} \geq 0 \\ 0 & \text { for } \lambda_{f}<0\end{cases}$

Based on previous parameter identification for native porcine aortic valve tissue, the parameters $k_{1}$ and $k_{2}$ are set to $0.7 \mathrm{kPa}$ and 9.9 , respectively (Billiar and Sacks 2000b; Driessen et al. 2005b). In addition, $G=$ $50 \mathrm{kPa}$ and $\phi_{\mathrm{tot}}=0.5$. The directions $\vec{v}_{1}$ and $\vec{v}_{2}$ are obtained from the principal loading directions in a leaflet with isotropic mechanical properties (Driessen et al. 2005b). The direction $\vec{v}_{1}$ is chosen to coincide with the major principal loading direction and $\vec{v}_{2}$ is subsequently constructed orthogonal to $\vec{v}_{1}$ in the plane of the leaflet using the normal $(\vec{n})$ of the leaflet (i.e., $\vec{v}_{2}=\vec{v}_{1} \times \vec{n}$ ). 
Fig. 3 Finite element meshes of the arterial wall a and aortic valve b. By symmetry, only one quarter of the tube and one half of a leaflet are modeled. These modeled parts of the arterial wall and aortic valve are discretized with 100 and 147 hexahedral elements, respectively
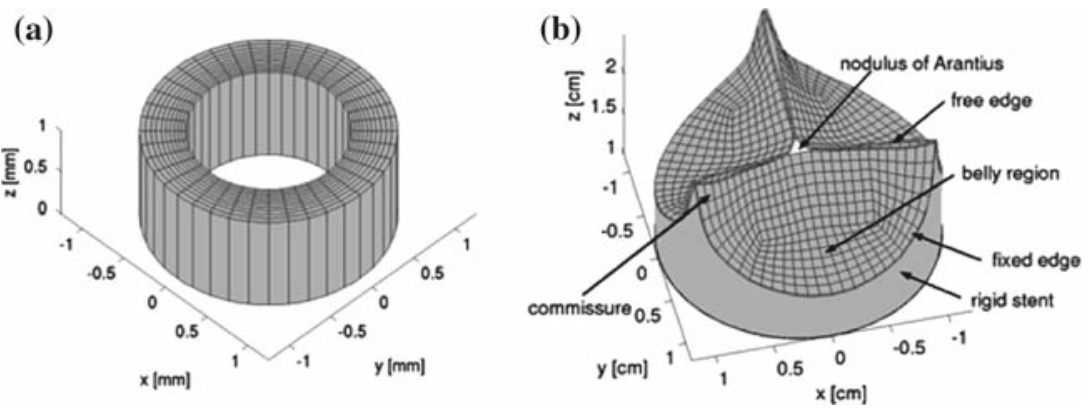

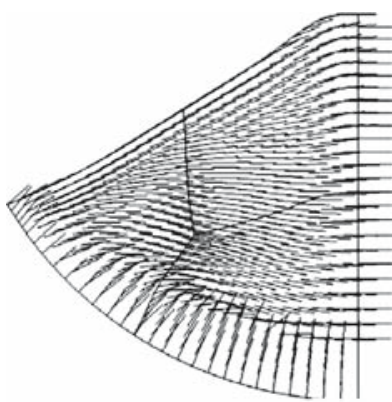

$\vec{v}_{1}$ ('circumferential')

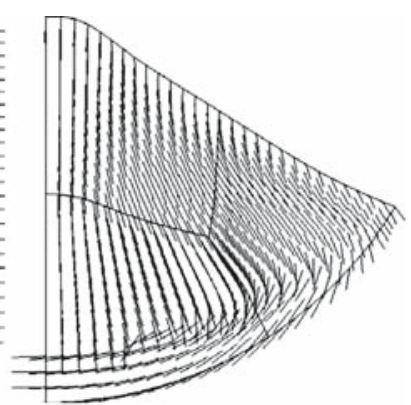

$\vec{v}_{2}$ ('radial')
Fig. 4 Local directions $\vec{v}_{1}$ (left) and $\vec{v}_{2}$ (right) in the undeformed configuration of the aortic heart valve leaflet, which are used to define the angular fiber distributions

Note that $\vec{v}_{1}$ basically coincides with the circumferential direction of the aortic valve leaflet, whereas $\vec{v}_{2}$ corresponds to the radial direction (Fig. 4).

\subsection{Remodelling}

In order to specify the stimulus functions $g_{i}(i=1,2)$ in Eqs. (8) and (9), both a strain-based and a stress-based approach are adopted. For the strain-based remodelling algorithm the stimulus functions are set equal to the strains $\left(\epsilon_{i}\right)$ in the direction of $\vec{v}_{i}$ :

$g_{i}= \begin{cases}\epsilon_{i}=\lambda_{i}-1=\sqrt{\vec{v}_{i} \cdot \boldsymbol{C} \cdot \vec{v}_{i}}-1 & \text { for } \epsilon_{i} \geq 0 \\ 0 & \text { for } \epsilon_{i}<0\end{cases}$

For the stress-based approach, on the other hand, the stimulus functions are set equal to the extra stresses $\left(\tau_{i}\right)$ in the direction of $\vec{v}_{i}$ :

$g_{i}= \begin{cases}\tau_{i}=\vec{v}_{i}^{\text {def }} \cdot \boldsymbol{\tau} \cdot \vec{v}_{i}^{\text {def }} & \text { for } \tau_{i} \geq 0 \\ 0 & \text { for } \tau_{i}<0\end{cases}$

where $\vec{v}_{i}^{\text {def }}$ denotes the direction of $\vec{v}_{i}$ in the deformed configuration. Equations (8), (9), (16) and (17) imply that for a uniaxial loading condition $\left(g_{1} \neq 0\right.$ and $g_{2}=0$ or $g_{1}=0$ and $g_{2} \neq 0$ ), all fiber directions align with the major loading direction $\left(\alpha_{p}=0^{\circ}, \beta_{p}=0\right)$. In case of a biaxial loading condition $\left(g_{1} \neq 0, g_{2} \neq 0\right)$, the two fiber families are situated in between $\vec{v}_{1}$ and $\vec{v}_{2}$ with the main orientation and dispersity $\left(\alpha_{p} \neq 0^{\circ}, \beta_{p} \neq 0\right)$ depending on the ratio of the stimulus functions. And for an equibiaxial loading condition $\left(g_{1}=g_{2}\right)$, the fibers are distributed uniformly $\left(\beta_{p} \rightarrow \infty\right)$ in the plane of $\vec{v}_{1}$ and $\vec{v}_{2}$. The parameter $k$ that controls the value of the preferred dispersity of the fiber distribution function in Eq. (9) is arbitrarily set to 1.0. Time is scaled with $\tau=1 \mathrm{~s}$ and the values of $\tau_{a} / \tau$ and $\tau_{b} / \tau$ are both set to 1.0. To prevent numerical problems with the expression for $\beta_{p}$ in Eq. (9), the maximum and minimum values of the preferred dispersity are set to 100 and 0.01 , respectively. These maximum and minimum values represent a uniform and uniaxial fiber distribution.

\subsection{Analyses and parameter variations}

To start with isotropic mechanical properties, a uniform fiber distribution (with initial values for $\alpha$ and $\beta$ of $45^{\circ}$ and 100 , respectively) is used. After the pressures have been applied, the remodelling algorithm is applied until the steady-state values of the fiber distribution parameters are obtained. Both the results of a strain-based (Eq. (16)) and a stress-based (Eq. (17)) remodelling algorithm are addressed. For the arterial wall, the value of $k$ is changed from 1.0 to 5.0 to study the effects of an increased dispersity, and $\lambda_{z}$ is increased from 1.2 to 1.6 to simulate the effects of an increased axial pre-stretch (Table 1). For the aortic valve, $G$ is decreased from 50 to $25 \mathrm{kPa}$ to show the consequences of a reduced matrix shear modulus (Table 2).

\section{Results}

\subsection{Arterial wall}

The predicted main fiber angle $\alpha$ and dispersity $\beta$ of the fiber distribution after the remodelling process as a function of the radial position in the arterial wall are shown in Fig. 5. The results obtained with the reference values of the parameters from Table 1 (case 1a) indicate that the main fiber angle as well as the dispersity increase 
Table 1 Overview of the performed analys for the arterial wall

\begin{tabular}{llll}
\hline Case & Stimulus & Dispersity parameter $k$ & Axial pre-stretch $\lambda_{z}$ \\
\hline $1 \mathrm{a}$ & Strain & 1.0 & 1.2 \\
$1 \mathrm{~b}$ & Stress & - & - \\
$1 \mathrm{c}$ & - & 5.0 & - \\
$1 \mathrm{~d}$ & - & - & 1.6 \\
\hline
\end{tabular}

A - indicates that the parameter values are equal to their reference values (case 1a)

Table 2 Overview of the performed analys for the aortic valve

\begin{tabular}{lll}
\hline Case & Stimulus & Matrix shear modulus $G(\mathrm{kPa})$ \\
\hline $2 \mathrm{a}$ & Strain & 50.0 \\
$2 \mathrm{~b}$ & Stress & - \\
$\mathrm{2c}$ & - & 25.0 \\
\hline
\end{tabular}

$\overline{\mathrm{A}-\text { indicates that the parameter values are equal to their refe- }}$ rence values (case $2 \mathrm{a}$ )

from the inner wall towards the outer wall. This is caused by the fact that the circumferential strains decrease as a function of the radial position, whereas the axial strain remains constant (due to $\lambda_{z}$ ). In other words, the ratio of the stimulus functions $g_{1} / g_{2}$ decreases from the inside to the outside. The corresponding angular fiber distributions at the inner wall $\left(\alpha= \pm 16^{\circ}\right.$ and $\left.\beta=0.4\right)$ and at the outer wall $\left(\alpha= \pm 34^{\circ}\right.$ and $\left.\beta=2.0\right)$ are shown in Fig. 6. The predicted main fiber directions at $\gamma= \pm \alpha$ represent two helically arranged families of fibers, oriented in between the circumferential and axial direction. The main fiber angle shifts from a circumferential orientation $\left(\gamma=0^{\circ}\right)$ towards the axial direction $\left(\gamma= \pm 90^{\circ}\right)$. At the same time, the dispersity increases and the fiber distribution becomes more uniform or isotropic. Switching the stimulus functions from strain-based $\left(g_{i}=\lambda_{i}-1=\epsilon_{i}\right)$ to stress-based $\left(g_{i}=\tau_{i}\right)$ results in a strong decrease of $\alpha$ and $\beta$ (case 1b). This is caused by the fact that the ratio of circumferential and axial stresses is much larger than the ratio of the strains (i.e., $\tau_{1} / \tau_{2}>\epsilon_{1} / \epsilon_{2}$ ) due to the nonlinearity of the constitutive equations for the arterial wall. Increasing the dispersity parameter from 1.0 to 5.0 (case 1c) hardly affects the main angle $\alpha$, but clearly causes an increase in the values of the dispersity $\beta$. Increasing the axial pre-stretch $\lambda_{z}$ (case $1 \mathrm{~d}$ ) from 1.2 to 1.6, on the other hand, causes an increase of $\alpha$ and $\beta$. This increase in $\lambda_{z}$ causes the circumferential and axial strains to become closer to each other. Even at $r_{0}=$ $0.80 \mathrm{~mm}$ they become equal and as a result $\alpha$ becomes $45^{\circ}$ and $\beta$ becomes very large $(\beta \rightarrow \infty)$. Thereafter, the axial strains are larger than the circumferential strains and, consequently, $\alpha$ is larger than $45^{\circ}$ and the dispersity $\beta$ decreases.

\subsection{Aortic valve}

In the commissure region and near the free edge, the leaflet is mainly loaded uniaxially. Consequently, the main fiber angle $\alpha$ and the dispersity $\beta$ become zero (Fig. 7a). This represents a uniaxial fiber distribution with locally all fibers oriented in the same direction. In the belly region, on the other hand, the leaflet is loaded biaxially which results in values for $\alpha$ and $\beta$ unequal to zero. The corresponding fiber architecture is shown in Fig. 8. In the uniaxially loaded regions, the two main fiber families coincide and consequently only one fiber direction can be distinguished. Toward the belly region of the leaflet, the two fiber families start to diverge and two directions can be discriminated. These predicted directions represent a branching fiber architecture that resemble a hammock-type structure. Using a stressbased remodelling algorithm (case $2 b$ ) instead of strainbased, decreases the values of $\alpha$ and $\beta$ in the belly region of the leaflet (Fig. 7b). This is again caused by the nonlinear expression of the constitutive equation. I.e., for the stimulus functions in the belly region, the ratio of stresses is much larger than the ratio of strains. Decreasing the shear modulus of the matrix material (case 2c), on the other hand, increases the size of the biaxially loaded belly region. Consequently, this causes an increase in the values of $\alpha$ and $\beta$ (Fig. 7c). Decreasing the matrix shear modulus increases the anisotropic nature of the mechanical behavior of the leaflet and causes a more pronounced biaxial loading condition. This phenomenon was also reported in a previous study (Driessen et al. 2005a).

The evolutions of the main fiber angle and dispersity for a node in the belly region of the leaflet as a function of scaled time $(t / \tau)$ are shown in Fig. 9. The values at $t / \tau=0$ denote the initial values of $\alpha\left(=45^{\circ}\right)$ and $\beta$ $(=100)$. For the cases with the reference values (case $2 a)$ and the decreased shear modulus (case 2c), $\alpha$ first decreases but slightly increases thereafter towards a stable value (Fig. 9a). For the stress-based situation (case $2 b$ ), on the other hand, $\alpha$ decreases monotonically to reach its steady-state value. This is probably caused by a 'positive-feedback' character of the remodelling framework for the stress-based case. That is, rotating the fibers towards the direction with the highest stresses causes the stresses in this direction to increase which might cause a further increase of the amount of fibers in this direction, and so on. At this point, it is worth noting that this process might be unstable and is further addressed in Sect. 4. The dispersities $\beta$ decrease monotonically to their steady-state values for all the three cases considered (Fig. 9b). 

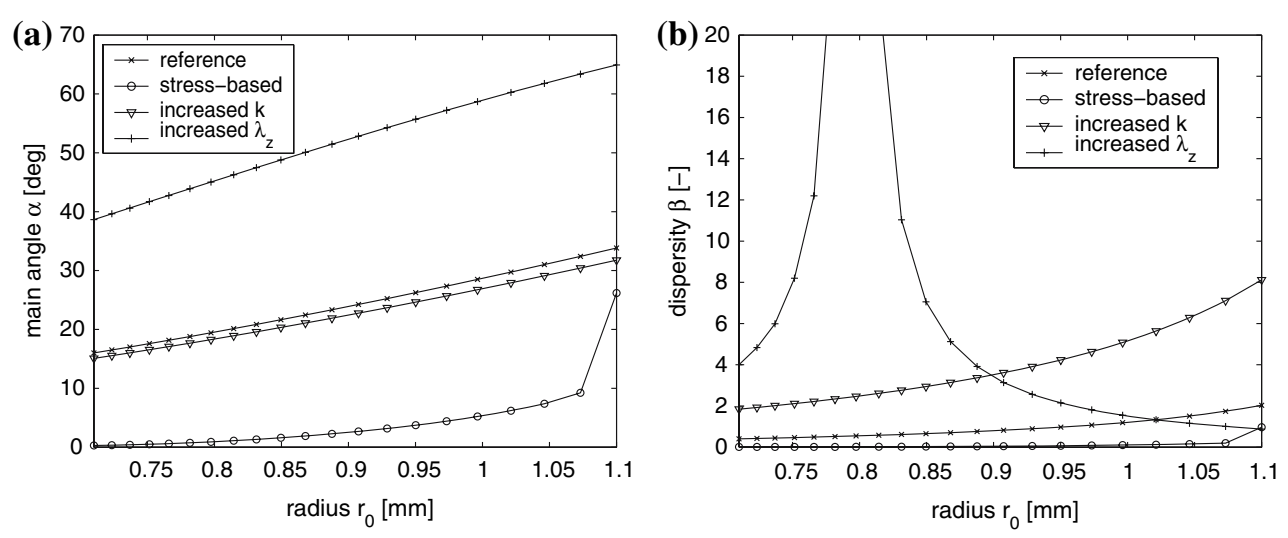

Fig. 5 Fiber distribution parameters as a function of the undeformed radius $r_{0}$ in the arterial wall. a Main fiber angle $\alpha$ [deg] and $\mathbf{b}$ dispersity $\beta[-]$. Note that the results in $\mathbf{b}$ are truncated to obtain a clearer representation of the data

Fig. 6 Representation of the angular fiber distributions at the inner $\mathbf{a}$ and outer $\mathbf{b}$ side of the arterial wall, obtained with reference values of the parameters (case 1a). $\gamma$ denotes the in-plane angle with $\gamma=0^{\circ}$ and $\gamma= \pm 90^{\circ}$ corresponding to the circumferential and axial direction, respectively
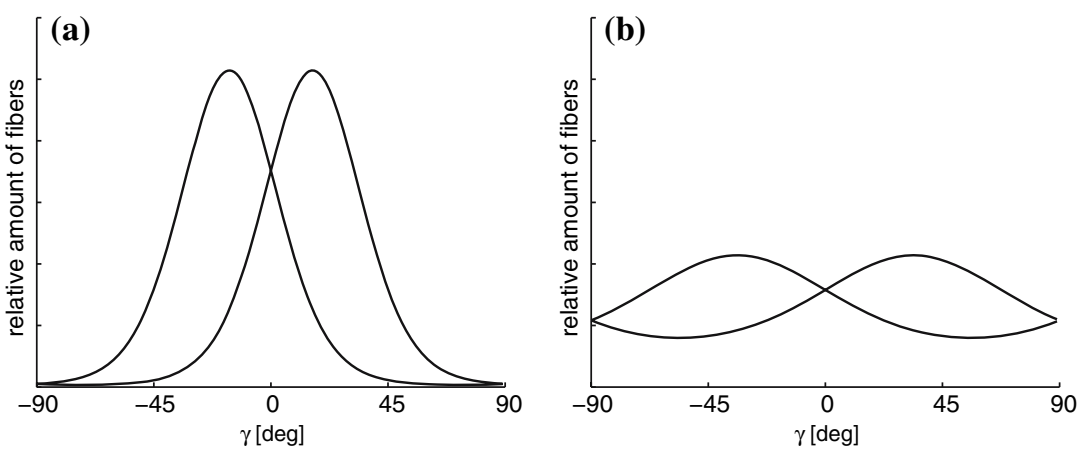

\section{Discussion}

In the present study, a computational framework is presented to investigate mechanically induced remodelling of the angular collagen fiber distribution in cardiovascular tissues. A structurally based constitutive model is employed that contains parameters to incorporate the collagen fiber architecture (Driessen et al. 2005b) and is extended with remodelling equations to account for changes in the fiber architecture (Driessen et al. 2004, 2005a). The model contains two structural parameters to describe the angular fiber distribution, i.e., the main fiber direction $\alpha$ and the dispersity $\beta$, and remodelling equations are defined to study mechanically induced changes in the fiber distribution. The model is applied to study collagen remodelling in the arterial wall and in the aortic valve, and strain-induced as well as stress-induced remodelling of the collagen architecture is addressed.

For the arterial wall, the model predictions show that the main fiber direction shifts from a circumferential orientation at the inner wall towards an axial orientation at the outer wall. In addition, the dispersity of the fiber distribution at the inside is larger than at the outside (Fig. 6). These observations agree to some extent with morphological measurements from litera- ture which reveal that the fibers in the media are circumferentially and coherently aligned, whereas in the adventitia, the pitch of the helically arranged fibers and the dispersity of the fiber distribution increase (Rhodin 1980; Canham et al. 1997; Finlay et al. 1995; Holzapfel et al. 2002; Gasser et al. 2006). In this study, however, the arterial wall is for simplicity modeled as a one-layer material, but obviously in future studies a two-layer structure (consisting of a media and adventitia) should be considered and the effects of residual stresses (modeled by an opening angle, see Fung 1990; Holzapfel et al. 2000) on the remodelling process could be incorporated. In that case, the distinct jump in fiber orientations between the media and adventitia of native vessels might be explained by employing different remodelling laws and reference configurations for the two layers of the arterial wall (Driessen et al. 2004).

For the aortic valve, the predicted fiber directions resemble a hammock-type fiber architecture with a uniaxial fiber distribution in the commissure region and a branching, diverging fiber architecture in the belly region of the leaflet (Fig. 8). These findings are also in accordance with observations from literature which reveal that the fibers in the aortic valve are mainly running in the circumferential direction from 

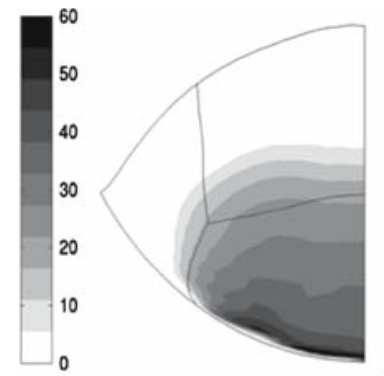

(a)
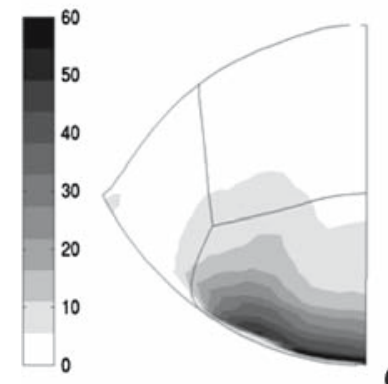

(b)
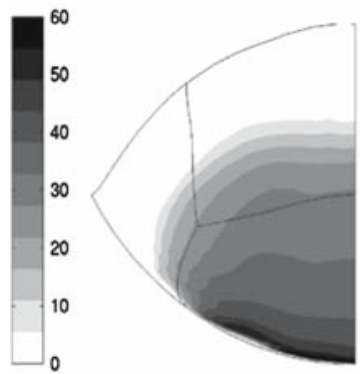

(c)

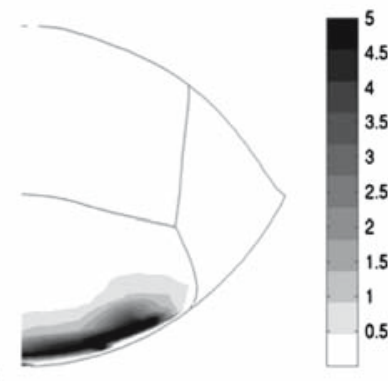

Fig. 7 Distribution of fiber parameters $\alpha$ [deg] (left) and $\beta[-]$ (right) in the aortic valve after remodelling. a is obtained with reference values of parameters (case $2 a$ ), b with a stress-based stimulus function (case $2 b$ ) and $\mathbf{c}$ with a reduced matrix shear modulus (case 2c)

commissure to commissure, whereas in the center more diverging bundles can be observed (Sauren 1981). In addition, the results are in accordance with Sacks et al. (1998) who have demonstrated that the collagen fibers in the commissure region are more aligned when compared to the belly region. A relatively high value for the shear modulus of the aortic valve matrix material is used to stabilize the finite element simulations and to prevent numerical problems. Further decreasing the matrix shear modulus will cause a more biaxial loading condition in the belly region of the leaflet, i.e., the ratio of radial and circumferential strains increases. It might even be possible that the radial strains become larger than the circumferential strains (Lo and Vesely 1995; Driessen et al. 2005b). In the context of the proposed remodelling framework in the present study, this would result in an unrealistic predicted fiber architecture

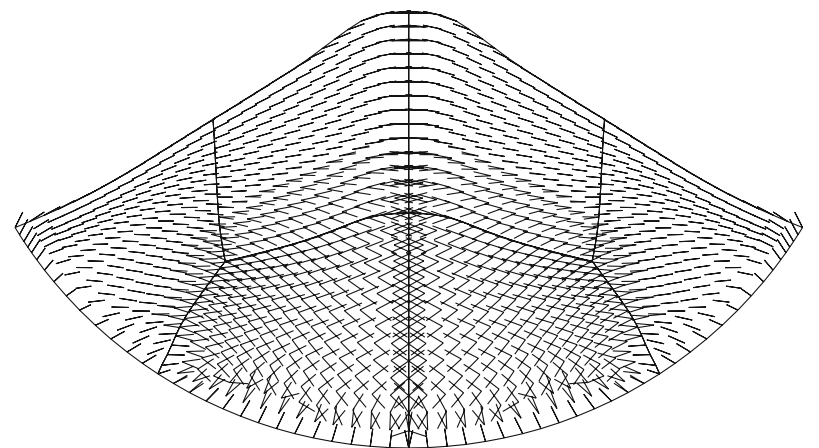

Fig. 8 Representation of the main fiber directions in the undeformed configuration of the aortic valve, obtained with the reference values of the parameters (case 2a). The main fiber directions are calculated from $\vec{e}_{f_{0}}=\cos (\alpha) \vec{v}_{1} \pm \sin (\alpha) \vec{v}_{2}$, with $\alpha$ the main fiber angle and $\vec{v}_{1}$ and $\vec{v}_{2}$ the local directions from Fig. 4

with more radially oriented instead of circumferentially oriented fibers. In that case, a stress-based remodelling algorithm would be more feasible because the circumferential stresses in the leaflet are larger than the radial stresses (Driessen et al. 2005b).

It is clear that at this point, only a qualitative comparison is made and that a quantitative comparison still needs to be assessed. Techniques that are based on small angle light scattering (Sacks et al. 1997), polarized light microscopy (Tower and Tranquillo 2001; Doehring et al. 2005) or confocal laser scanning microscopy (Brightman et al.2000) enable us to measure the fiber architecture in cardiovascular tissues and might be beneficial to further validate the remodelling framework. Some of the remodelling parameters (e.g., stimulus functions $g_{i}$ and $k$ ) are chosen more or less randomly to demonstrate the effects of these parameters on the predicted fiber architectures. Eventually these experimental techniques should be used to assess the model parameters quantitatively by comparing the measured and predicted fiber architecture.

Based on previous collagen remodelling studies (Driessen et al. 2004, 2005a; Wilson et al. 2006), we hypothesize that the main fiber directions are situated in between the principal loading directions. For the presented remodelling framework in this study, this assumption implies that the local directions $\left(\vec{v}_{1}\right.$ and $\left.\vec{v}_{2}\right)$ should coincide with the principal loading directions. This is the case for the arterial wall as the local directions and principal loading directions coincide with the circumferential and axial direction, and these do not change during the remodelling process. For the aortic valve, a deviation can be present, as the local directions are not always exactly aligned with the principal loading directions and these might change during remodelling. However, this deviation is relatively small (mean angular deviation $<10^{\circ}$ ) and it is expected that this does 
Fig. 9 Evolution of the fiber parameters for a node in the belly region of the valve leaflet as a function of scaled time. a Main fiber angle $\alpha$ [deg] and $\mathbf{b}$ dispersity $\beta[-]$. Note that the results in $\mathbf{b}$ are truncated to obtain a clearer representation of the data
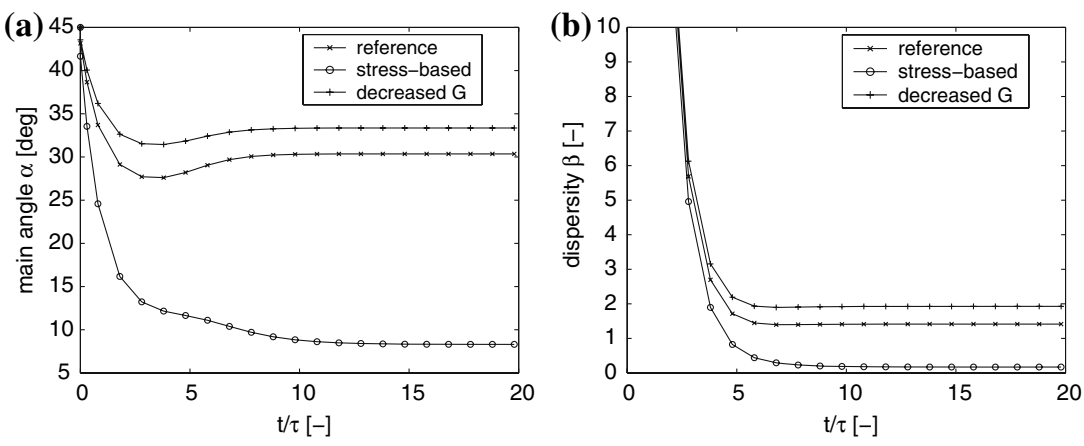

not affect the results significantly. A limitation of the model by Driessen et al. $(2004,2005 a)$ is its ambiguous behavior in (nearly) equibiaxial loading conditions, because in these cases the principal loading directions cannot be determined uniquely. As a consequence, the previous model predicted random preferred fiber directions that depended on the arbitrarily calculated principal loading directions. The present study overcomes this shortcoming by considering remodelling of the angular fiber distribution and predicts a uniform distribution in case of an equibiaxial loading condition. In addition, in previous collagen remodelling studies for the aortic valve (Driessen et al. 2003a, 2005a), the mechanical properties for the leaflet tissue were chosen more or less arbitrarily. However, in the current study, these parameters are obtained from literature based on experimental data.

In case of a deformation-controlled loading condition, the stress-based remodelling algorithm might be unstable due to a 'positive-feedback' mechanism present in the model. When the deformation is fixed, all fibers will eventually align with the direction that initially carries the highest stresses. For example, when the stresses are highest in the direction of $\vec{v}_{1}$, the fibers will rotate toward this direction and $\alpha$ decreases. This causes a further increase in the stresses in the direction of $\vec{v}_{1}$ which results in a further decrease of $\alpha$ and $\beta$, and so on. In this case, all fibers will eventually be aligned with $\vec{v}_{1}$ (i.e., $\alpha \rightarrow 0^{\circ}$ and $\beta \rightarrow 0$ ). This also implies that the final solution might depend on the initial fiber architecture. It can cause problems in case of a stress-based remodelling algorithm for the arterial wall because the axial pre-stretch $\left(\lambda_{z}\right)$ is fixed in the current model and we expect that applying an axial pre-stress might be more realistic in that case.

The strain-based and stress-based remodelling algorithms employed in this study both give reasonable and qualitatively comparable results, and the exact stimulus for remodelling remains presently unknown. Rodriguez et al. (1994) use stress as a growth stimulus and exclude strain as a possible stimulus because of the difficulty in defining a reference configuration in remodelling tissues. Cowin (1996) subsequently extends the theory of Rodriguez et al. (1994) to include the possibility of strain or rate of deformation as growth stimulus. Humphrey (2001) argues that cells cannot respond directly to the mathematical (not physical) concepts of strain or stress, but these concepts can be correlated with tissue remodelling using phenomenological relations or empirical correlations.

Although the model predictions agree qualitatively with observations from literature for native tissues, the mechanisms by which the cells remodel the collagen fiber architecture to adapt to changes in functional demands are not yet well understood. It is known that cells can reorganize the collagen fibers by reorientation and degradation of existing collagen fibers, and by synthesis and directed secretion of newly formed fibers (Nakatsuji and Johnson 1984; Birk and Trelstad 1984, 1986; Guidry and Grinnell 1985; Prajapati et al. 2000; MacKenna et al. 2000; Sawhney and Howard 2002). However, experiments have to be performed to further clarify the exact mechanisms of these processes and to determine their relative contributions to collagen reorganization. Experiments with well-defined loading conditions should be employed (Mol et al. 2003; Balestrini and Billiar 2006) and the feasibility of assessing collagen remodelling in life tissue cultures with real-time visualization techniques should be further addressed (Krahn et al. 2006).

We only describe changes in the collagen fiber distribution, but it might be desirable to also incorporate changes in collagen type, collagen content, fiber thickness and cross-linking into the model as these properties are also subjected to remodelling and play an important role in the mechanics of soft connective tissues (e.g., Doillon et al. 1985; Carver et al. 1991; Villarreal and Dillmann 1992; Dahl et al. 2005; Avery and Bailey 2005). Remodelling of the elastin architecture will also be of interest as this is an important contributor to the mechanics of cardiovascular tissues (Vesely 1998; Holzapfel et al. 2000) and could be a missing 
link in tissue engineering of cardiovascular constructs (Patel et al. 2006). Moreover, the arterial wall and aortic valve are loaded (quasi-) statically by assuming that the time constant of the remodelling process is much larger than the time constant of the pressure load. Consequently, the dynamic response of the tissues is not taken into account. Only the solid mechanics of the tissues is considered and any interaction with the surrounding fluid is omitted. Flow of the surrounding fluid induces shear stresses which are known to be important factors in cardiovascular remodelling (see, e.g., Humphrey 2002). Whereas shear stresses might be computed easily in a straight arterial segment, more complex fluidsolid interaction models might be necessary for heart valves (Einstein 2002; de Hart 2002). Finally, (volumetric) growth is not taken into account in the present study and, consequently, the volume and geometry of the tissues remain constant during remodelling. However, changes in geometry and the presence of residual stresses/strains (due to growth) might affect the mechanical loading condition and therefore the model predictions. Once these issues have been resolved, the presented model can be applied to further optimize the conditioning protocols and, consequently, the mechanical behavior of tissue-engineered arteries and heart valves (Niklason et al. 1999; Hoerstrup et al. 2000; Mol et al. 2006). Finally, we expect that the remodelling framework might be employed to incorporate collagen fiber orientations in complex geometries, such as arterial bifurcations (Hariton et al. 2005).

\section{References}

Avery NC, Bailey AJ (2005) Enzymic and non-enzymic crosslinking mechanisms in relation to turnover of collagen: relevance to aging and exercise. Scand J Med Sci Sports 15:231-240

Baek S, Rajagopal KR, Humphrey JD (2006) A theoretical model of enlarging intracranial fusiform aneurysms. J Biomech Eng 128:142-149

Balestrini JL, Billiar KL (2006) Equibiaxial cyclic stretch stimulates fibroblasts to rapidly remodel fibrin. J Biomech (in press). Available online

Bathe KJ (1996) Finite element procedures. Prentice Hall, Englewood Cliffs

Billiar KL, Sacks MS (2000a) Biaxial mechanical properties of the natural and glutaraldehyde treated aortic valve cusp-part I: experimental results. J Biomech Eng 122:23-30

Billiar KL, Sacks MS (2000b) Biaxial mechanical properties of the natural and glutaraldehyde treated aortic valve cusp-part II: a structural constitutive model. J Biomech Eng 122:327-335

Birk DE, Trelstad RL (1984) Extracellular compartments in matrix morphogenesis: collagen fibril, bundle, and lamellar formation by corneal fibroblasts. J Cell Biol 99:2024-2033

Birk DE, Trelstad RL (1986) Extracellular compartments in tendon morphogenesis: collagen fibril, bundle, and macroaggregate formation. J Cell Biol 103:231-240
Boerboom RA, Driessen NJB, Bouten CVC, Huyghe JM, Baaijens FPT (2003) Finite element model of mechanically induced collagen fiber synthesis and degradation in the aortic valve. Ann Biomed Eng 31:1040-1053

Brightman AO, Rajwa BP, Sturgis JE, McCallister ME, Robinson JP, Voytik-Harbin SL (2000) Time-laps confocal reflection microscopy of collagen fibrillogenesis and extracellular matrix assembly in vitro. Biopolymers 54: 222-234

Canham PB, Finlay HM, Boughner DR (1997) Contrasting structure of the saphenous vein and internal mammary artery used as coronary bypass vessels. Cardiovasc Res 34:557-567

Carver W, Nagpal ML, Nachtigal M, Borg TK, Terracio L (1991) Collagen expression in mechanically stimulated cardiac fibroblasts. Circulat Res 69:116-122

Cowin SC (1996) Strain or deformation rate dependent finite growth in soft tissues. J Biomech 29:647-649

Dahl SL, Rucker RB, Niklason LE (2005) Effects of copper and cross-linking on the extracellular matrix of tissue-engineered arteries. Cell Transpla 14:861-868

de Hart J (2002) Fluid-structure interaction in the aortic heart valve: a three-dimensional computational analysis. $\mathrm{PhD}$ thesis, Technische Universiteit Eindhoven

Doehring TC, Kahelin M, Vesely I (2005) Mesostructures of the aortic valve. J Heart Valve Disease 14:679-686

Doillon CJ, Dunn MG, Bender E, Silver FH (1985) Collagen fiber formation in repair tissue: development of strength and toughness. Collagen Relat Res 5:481-492

Driessen NJB, Boerboom RA, Huyghe JM, Bouten CVC, Baaijens FPT (2003a) Computational analyses of mechanically induced collagen fiber remodelling in the aortic heart valve. J Biomech Eng 125:549-557

Driessen NJB, Peters GWM, Huyghe JMRJ, Bouten CVC, Baaijens FPT (2003b) Remodelling of continuously distributed collagen fibres in soft connective tissues. J Biomech 36:1151-1158

Driessen NJB, Wilson W, Bouten CVC, Baaijens FPT (2004) A computational model for collagen fibre remodelling in the arterial wall. J Theor Biol 226:53-64

Driessen NJB, Bouten CVC, Baaijens FPT (2005a) Improved prediction of the collagen fiber architecture in the aortic heart valve. J Biomech Eng 127:329-336

Driessen NJB, Bouten CVC, Baaijens FPT (2005b) A structural constitutive model for collagenous cardiovascular tissue incorporating the angular fiber distribution. J. Biomech Eng 127: 494-503

Driessen NJB, Mol A, Bouten CVC, Baaijens FPT (2007) Modeling the mechanics of tissue-engineered human heart valve leaflets. J Biomech 40:325-334

Einstein DR (2002) Nonlinear acoustic analysis of the mitral valve. $\mathrm{PhD}$ thesis, University of Washington, Seattle

Finlay HM, McCullough L, Canham PB (1995) Three-dimensional collagen organization of human brain arteries at different transmural pressures. J Vasc Res 32:301-312

Fung YC (1990) Biomechanics: motion, flow, stress and growth. Springer, New York

Gasser TC, Ogden RW, Holzapfel GA (2006) Hyperelastic modelling of arterial layers with distributed collagen fibre orientations. J Roy Soc Interface 3:15-35

Guidry C, Grinnell F (1985) Studies on the mechanism of hydrated collagen gel reorganization by human skin fibroblasts. J Cell Sci 79:67-81

Hariton I, deBotton, G, Gasser TC, Holzapfel GA (2005) How to incorporate collagen fiber orientations in an arterial bifurcation. In: Hamza MH (ed) Proceedings of the 3rd IASTED international conference on biomechanics. Acta Press, Anaheim 
Hariton I, Debotton G, Gasser TC, Holzapfel GA (2006) Stressdriven collagen fiber remodelling in arterial walls. Biomech Model Mechanobiol (in press). Available online

Hoerstrup SP, Sodian R, Daebritz S, Wang J, Bacha EA, Martin DP, Moran AM, Guleresian J, Sperling JS, Kaushal S, Vacanti JP, Schoen FJ, Mayer JE (2000) Functional living trileaflet heart valves grown in vitro. Circulation 102:III49III49

Holzapfel GA, Gasser TC, Ogden RW (2000) A new constitutive framework for arterial wall mechanics and a comparative study of material models. J Elast 61:1-48

Holzapfel GA, Gasser TC, Stadler M (2002) A structural model for the viscoelastic behavior of arterial walls: Continuum formulation and finite element analysis. Euro J Mech A Solids 21:441-463

Humphrey JD (1999) Remodelling of a collagenous tissue at fixed lengths. J Biomech Eng 121:591-597

Humphrey JD (2001) Stress, strain, and mechanotransduction in cells. J. Biomech Eng 123:638-641

Humphrey JD (2002) Cardiovascular solid mechanics: cells, tissues, and organs. Springer, New York

Krahn KN, Bouten CVC, van Tuijl S, van Zandvoort MAMJ, Merkx M (2006) Fluorecently labeled collagen binding proteins allow specific visualization of collagen in tissues and live cell culture. Anal Biochem 350:177-185

Lo D, Vesely I (1995) Biaxial strain analysis of the porcine aortic valve. Ann Thorac Surg 60:S374-378

MacKenna D, Summerour SR, Villarreal FJ (2000) Role of mechanical factors in modulating cardiac fibroblast function and extracellular matrix synthesis. Cardiovasc Res 46:257-263

Mol A, Bouten CVC, Zund G, Guenter CI, Visjager JF, Turina M, Baaijens, FPT, Hoerstrup SP (2003) The relevance of large strains in functional tissue engineering of heart valves. Thorac Cardiovasc Surg 51:78-83

Mol A, Rutten MCM, Driessen NJB, Bouten CVC, Zund G, Baaijens FPT, Hoerstrup SP (2006) Autologous human tissueengineered heart valves: prospects for systemic applications. Circulation 114:I152-I158

Nakatsuji N, Johnson KE (1984) Experimental manipulation of a contact guidance system in amphibian gastrulation by mechanical tension. Nature 307:453-455

Niklason LE, Gao J, Abbott WM, Hirschi KK, Houser S, Marini R, Langer R (1999) Functional arteries grown in vitro. Science 284:489-493
Olsen L, Maini PK, Sherratt JA, and Dallon JC (1999) Mathematical modelling of anisotropy in fibrous connective tissue. Math Biosci 158:145-170

Patel A, Fine B, Sandig M, Mequanint K. (2006) Elastin biosynthesis: The missing link in tissue-engineered blood vessels. Cardiovasc Res 71:40-49

Prajapati RT, Chavally-Mis B, Herbage D, Eastwood M, Brown RA (2000) Mechanical loading regulates protease production by fibroblasts in three-dimensional collagen substrates. Wound Repair Regenerat 8:226-237

Rhodin JAG (1980) Architecture of the vessel wall. In: Sparks HV Jr, Bohr DF, Somlyo AD, Geiger SR (eds) Handbook of physiology, The cardiovascular system, vol 2. American Physiological Society, Bethesda, pp. 1-31

Rodriguez EK, Hoger A, McCulloch AD (1994) Stress-dependent finite growth in soft elastic tissues. J Biomech 27:455-467

Sacks MS, Smith DB, Hiester ED (1997) A small angle light scattering device for planar connective tissue microstructural analysis. Ann Biomed Eng 25:678-689

Sacks MS, Smith DB, Hiester ED (1998) The aortic valve microstructure: effects of transvalvular pressure. J Biomed Mater Res 41:131-141

Sauren AAHJ (1981) The mechanical behaviour of the aortic valve. $\mathrm{PhD}$ thesis, Technische Hogeschool Eindhoven

Sawhney RK, Howard J (2002). Slow local movements of collagen fibers by fibroblasts drive the rapid global self-organization of collagen gels. J Cell Biol 157:1083-1091

Segal A (1984) SEPRAN user manual, standard problems and programmers guide. Leidschendam: Ingenieursbureau SEPRA, the Netherlands

Tower TT, Tranquillo RT (2001) Alignment maps of tissues: II. fast harmonic analysis for imaging. Biophys J 81:2964-2971

Vesely I (1998) The role of elastin in aortic valve mechanics. J Biomech 31:115-123

Villarreal FJ, Dillmann WH (1992) Cardiac hypertrophy-induced changes in mRNA levels for TGF-beta1, fibronectin, and collagen. Am J Physiol 262:H1861-H1866

Wilson W, Driessen NJB, van Donkelaar CC, Ito K (2006) Mechanoregulation of collagen orientation in articular cartilage. Osteoarthr Cartil 14:1196-1202 